\title{
Development of ultrasonic thickness measurements of solidification front inside metallurgical reactors
}

\author{
Jimmy Floquet ${ }^{1}$, Martin Désilets $^{2, a}$, Philippe Micheau ${ }^{1}$ And Nicolas Quaegebeur ${ }^{1}$ \\ 1 Mechanical Engineering Department, Université de Sherbrooke, Sherbrooke, Québec, J1K 2R1, Canada \\ 2 Chemical Engineering Department, Université de Sherbrooke, Sherbrooke, Québec, J1K 2R1, Canada
}

Received 11 April 2014, Accepted 13 August 2014

\begin{abstract}
The corrosive molten salts used for aluminum electrolysis attack the walls of the electrochemical reactors, reducing their lifetime and increasing production costs. Fortunately, a ledge is formed on those walls by the solidification of the molten salts used as the electrolytic bath, due to the heat losses through the sides of the reactors. This ledge is essential, protecting the sidewalls from the corrosive effect of the bath. Its thickness must however be controlled to avoid a reduction of the efficiency caused by a partial reduction of the electrolysis surface. One challenge is to measure the ledge thickness inside operating cells. The originality of this work resides in the development of an ultrasonic device to provide a non-intrusive ledge thickness measurement. Experimental results are obtained on a specially developed setup using the proposed ultrasonic device and are compared to ledge thicknesses obtained with an intrusive probe. The ultrasonic thickness measurement concept is validated up to $950{ }^{\circ} \mathrm{C}$ with a satisfactory accuracy inside $6 \mathrm{~mm}$ of the intrusive measurement.
\end{abstract}

Key words: Ultrasound / NDT / high temperature / aluminum / electrolysis cell

\section{Introduction}

The development of the metallurgical industry can be hindered by the important increase of energy and maintenance costs. In aluminum electrolysis cells, one of the most important factors is cell lifetime, which typically doesn't exceed 2500 days for a cell operated in normal conditions [1]. In a typical plant, where a cell is replaced every 6 days, the replacement cost for a cell, including losses of production and labor, is of the order of hundreds of thousands dollars. Thus, increasing the cell lifetime is an important issue for this industry. However, decreasing maintenance and replacement costs represent a challenge that cannot be aimed regardless of the productivity aspects.

Yet, the aluminum electrolysis represents a complex chemo-thermo-electro-mechanical process, involving many operational and design parameters. During the regular operation of a cell, the electrolytic bath acts as a phase change material (PCM) and solidifies on its sidewalls due to an important heat flux, intentionally held to maintain the thermal equilibrium between the cell and its external environment. More precisely, the sidewall design and the operating conditions are selected in order to keep

\footnotetext{
${ }^{a}$ Corresponding author:

Martin.Desilets@USherbrooke.ca
}

a frozen PCM layer on the sidewalls, despite a consequent heat generation effect induced by the passage of several thousands of amperes through the electrolyte. This frozen layer of PCM, called the ledge, is in fact protecting the cell from the corrosive action of the high temperature bath, typically held around $950{ }^{\circ} \mathrm{C}$ during regular operation. Maintaining its thickness to an appropriate target is one of the key factors to improve the cell life while maintaining optimal conditions for the production of aluminum.

The impact of the ledge on the cell lifetime has been widely studied. Stevens [2] has shown that a cell directly exposed to liquid electrolyte presents a critical failure after approximately 77 days against 2500 days for cells adequately protected by the ledge. Based on this study, the corrosion rate of the sidewall is extremely fast $(1.3 \mathrm{~mm}$ a day), illustrating the crucial role of the ledge. The degradation of the cell sidewall can lead to its rupture and catastrophic spill, an industrial risk to avoid. Moreover, the energy efficiency of the process highly depends on the ledge, the heat losses being directly related to the thickness of the ledge [3]. The important technological challenge is to perform a realistic and precise ledge thickness measurement, without disturbing the operation of the cell, and in an extremely hostile environment (temperatures between 300 and $1000{ }^{\circ} \mathrm{C}$, dust, magnetic fields, corrosive PCM). 


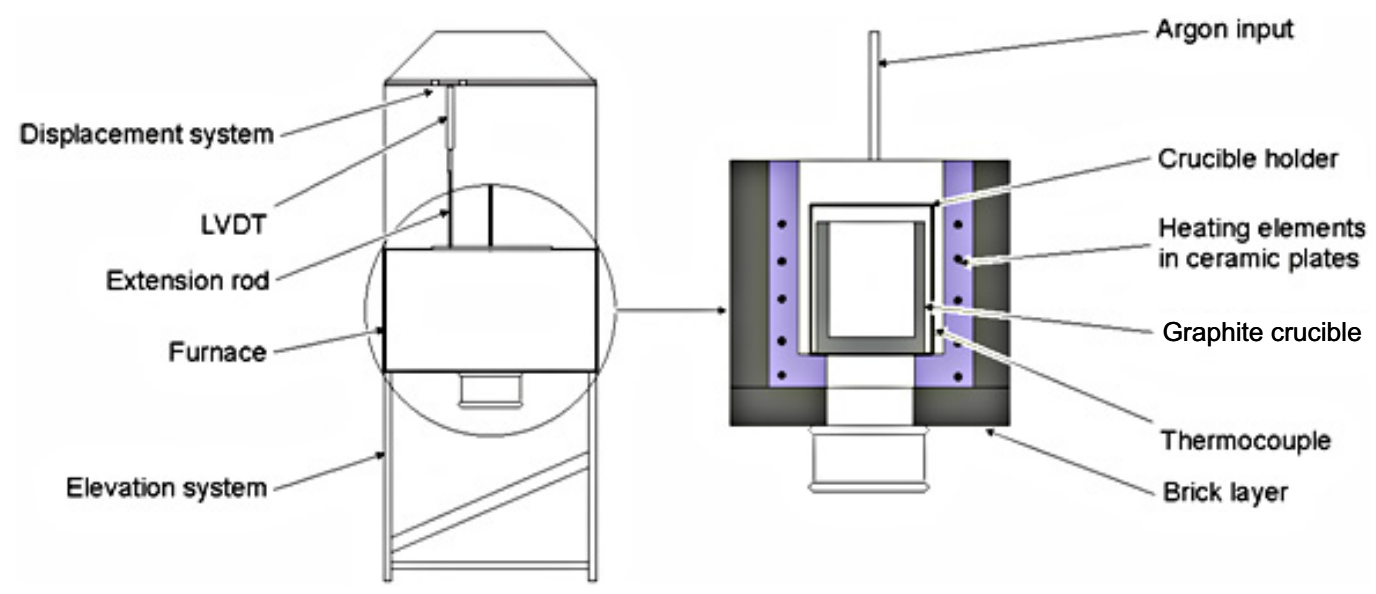

Fig. 1. Experimental setup (left) with details on the crucible environment (right).

Intrusive measurements, using a probe to palpate the ledge profile, are typically used to determine the position of such a solidification front. These techniques are usually risky, time-consuming and very intrusive. After such measurements, it takes days before the cell establishes a new thermal equilibrium. Qualified personnel is also required in order to conduct this procedure, unless an automatic system can be used [4]. Consequently, in this context, it is almost impossible to have a continuous solidification profile of the ledge in real-time using such an approach.

Bertrand et al. $[5,6]$ proposed a technique based on predictive measurements of the PCM taken from the exterior conditions of a metallurgical reactor. In this case, an infrared camera has been chosen as the external measurement technique to feed a predictive inverse method, as reported by Marois et al. [6,7]. The predictive indirect technique (inverse method) and direct measurements (LVDT coupled to an extension rod) have been compared on a system where a molten salt is solidified on a cooled surface. Results showed that it is possible to predict the PCM thickness evolution with the inverse method based on external thermal measurements. However, this technique is time-consuming and requires a reliable numerical model of the heat transfer problem occurring in the cell.

In similar industrial problems, thickness measurements are usually performed by measuring the Time-ofFlight of ultrasonic (US) waves through the materials. The ultrasonic waves can be generated and measured using contact or non-contact probes. Among existing noncontact emitter transducers, laser US excitation [8,9], electro magnetic acoustic transducer (EMAT) [10] or aircoupled probes [11] are limited in terms of the transmitted energy to the structure, such that thickness measurements in highly damped materials are impossible and contact transducers are usually required. Classical ultrasonic wall thickness contact probes are based on piezoceramic transducers used in compression mode [12], in pulse-echo or angular configurations $[13,14]$. To avoid any detrimental effects due to high temperature surfaces, a delay line material (wedge) is used as thermal insulation between the active transducer element and the test surface to limit the temperature increase of the transducer and avoid depolarization of the piezoceramic elements [15]. Cegla et al. [16] determined that such a delay line material (waveguide) avoids any failure of conventional ultrasonic transducers, during 4 weeks, so as to monitor metallic wall thicknesses.

The main objective of this study is to perform the ledge measurement by an US method. The present work aims at showing the feasibility of a non-intrusive measurement of the ledge thickness, conducted in an environment mimicing the behaviour of a real aluminum electrolysis cell. More precisely, the authors are proposing a new US sensor giving repeatable and safe measurements of the ledge thickness, at a solid/liquid interface, found inside metallurgical reactors.

First, the experimental setup and US device are described along with the measurement procedure. Then, experimental thickness measurements are performed on a setup where real industrial molten salts are frozen over a cooled surface. The accuracy of the estimated thicknesses is assessed by a comparison of the US measurements with intrusive direct measurements obtained via a LVDT sensor. A preliminary statistical analysis is then presented to assess the validity of the measurement and the concept developed. Finally, the possible improvements and future developments are outlined.

\section{Experimental setup}

The experimental setup used in this study is based on the one described and developed by Bertrand et al. [5]. Figure 1 shows a brief description of the facilities.

As suggested in reference [5], an opening is made at the bottom of the oven so as to expose one surface of the crucible to the cooling system, injecting a constant inert gas flow, in order to solidify the PCM. The opening also allows positioning of the US thickness measurement device. Figure 2 illustrates the concept of the proposed US device and the measurement paths and Figure 3 presents a photograph of the device. 


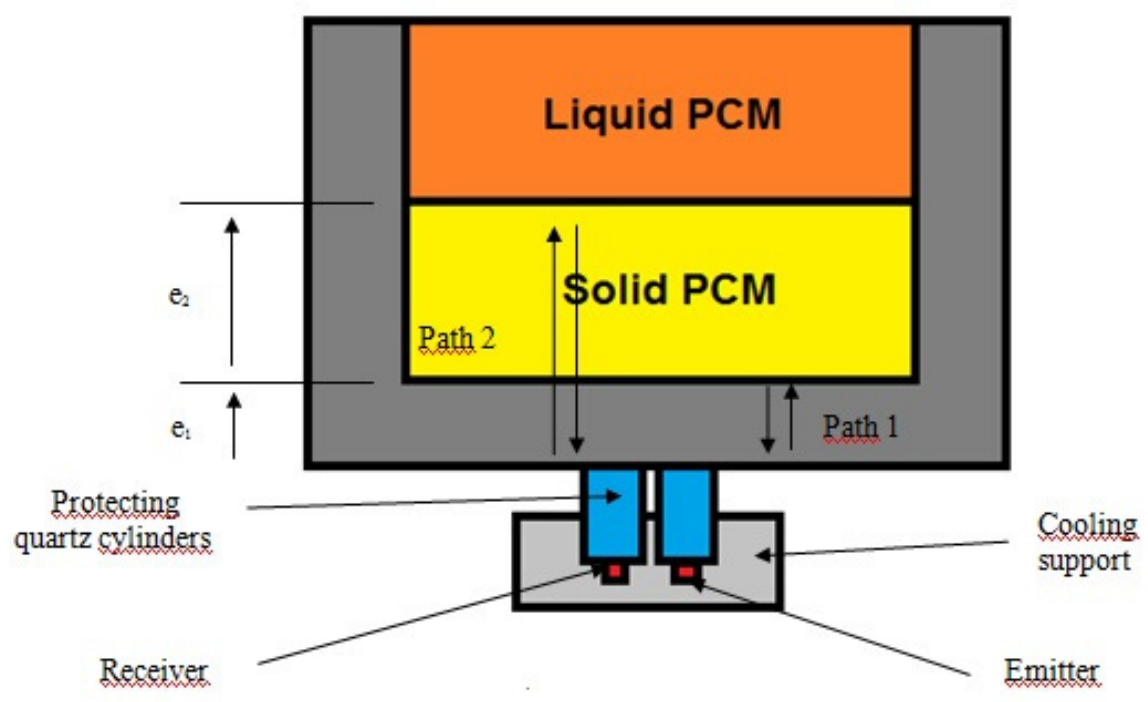

Fig. 2. Concept of the US device measurement.

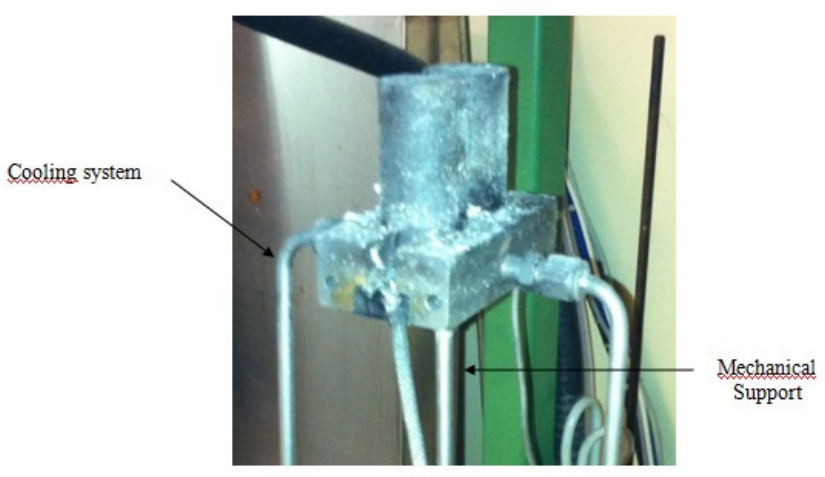

Fig. 3. Photography of the ultrasonic device, positioned on its support.

The US device is composed of two separate transducers: a 5-mm diameter piezoceramic (PZT 5A, PIC 255, Physik Instrumente GmbH and Co. KG, Karlsruhe, Germany) used as an actuator and an acoustic emission (AE) sensor (D9215, Mistras Group, Physical Acoustics Corporation, Princeton, USA), designed to receive the US signal. Each transducer is mounted on a quartz cylinder, acting both as a thermal insulator and as an ultrasonic impedance adaptator. This assembly is positioned inside a support, as illustrated in Figure 2. This support is perforated in order to introduce a cooling inert gas flow. This whole system aims at limiting the time exposure of the actuator to high temperature, in order to keep the piezoceramic as far as possible from its Curie temperature (approximately $350{ }^{\circ} \mathrm{C}$ ). A waveform generator (33120A, Agilent Technologies Inc., Santa Clara, CA) combined with a high-voltage amplifier (UA-8400, Musilab Inc., Drummondville, QC, Canada) are used to generate Hanning windowed sinusoidal burst (Fig. 4). The measurement of AE sensor is performed using a high impedance

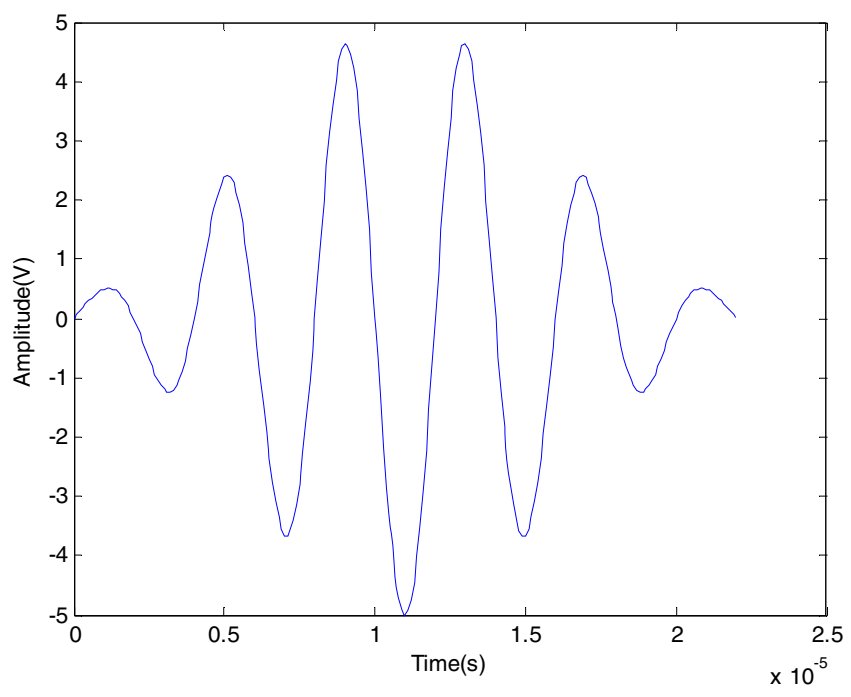

Fig. 4. Example of input signal (5.5 cycles Hanning windowed burst with a central frequency of $250 \mathrm{kHz}$ ).

N.I. PCI-5105 12-bit acquisition board (National Instruments Corp) via a custom LabVIEW interface. A typical signal received is presented in Figure 5.

The US device is positioned at the bottom of the oven, directly on the carbon crucible, in order to conduct the measurement. A mechanical displacement system using spring components is used to guarantee a constant and repeatable contact between the probes and the surface. Due to high temperature $\left(300{ }^{\circ} \mathrm{C}<T<1000{ }^{\circ} \mathrm{C}\right)$, contact must be avoided during non-testing period. A thermocouple is also positioned inside crucible to monitor the temperature of the PCM during the cooling sequence.

The ultrasonic thickness measurement is based on the detection of the echoes in the received signal, as shown in Figure 5 [14]. The first echo (due to the path 1 as 


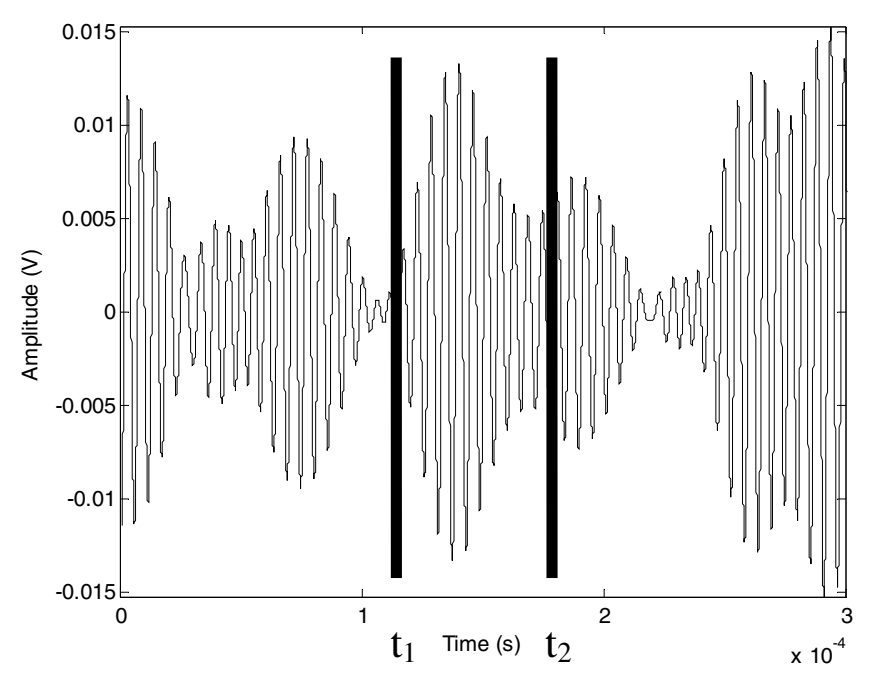

Fig. 5. Typical signal received at $1000{ }^{\circ} \mathrm{C}$. The Time-of-Flight of the two first echoes is indicated using bold black lines.

shown in Fig. 2) is induced by the boundary between the crucible and the ledge. Hence, the crucible thickness is estimated as:

$$
e_{1}=c_{1} \frac{t_{1}}{2}
$$

where $c_{1}=2130 \mathrm{~m} . \mathrm{s}^{-1}$ is the group velocity of the pressure wave in the graphite, and $t_{1}$ is the Time-of-Flight of the pressure wave along the first path.

The second echo (due to the path 2 as shown in Fig. 2) is due to the detection of the interface between the two phases of the PCM. The PCM layer thickness is estimated as:

$$
e_{2}=\frac{c_{2}\left(t_{2}-t_{1}\right)}{2}
$$

where $c_{2}=1280 \mathrm{~m} . \mathrm{s}^{-1}$ is the group velocity of the pressure wave of solid PCM, and $t_{2}$ is the Time-of-Flight of the pressure wave along the second path.

The quartz thickness is known and it is characterized acoustically. Because the Time-of-Flight is known and invariable in this material, it is simply removed from the measurement to determine the Time-of-Flight in both materials studied.

\section{Experimental procedure}

The experimental PCM is an industrial molten salt, in solid state at ambient conditions, used in the production of aluminum. The PCM has to be in liquid state during the operation of the experimental setup, so as to create an environment as close as possible to the industrial application. Its composition can be approximated by the following chemical composition: $80.5 \%$ of cryolite $\left(\mathrm{Na}_{3} \mathrm{AlF}_{6}\right)$, $11.5 \%$ of $\mathrm{AlF}_{3}, 5.5 \%$ of $\mathrm{CaF}_{2}$ and $2.5 \%$ of $\mathrm{Al}_{2} \mathrm{O}_{3}$. In order to mimic realistic operating conditions, the following experimental procedure is used:

1. The operating furnace temperature is set to $1000{ }^{\circ} \mathrm{C}$ (the PCM melts around $950^{\circ} \mathrm{C}$ ) with an argon volumetric flow rate of $34 \mathrm{~L} . \mathrm{min}^{-1}$ (50 psi) inside crucible

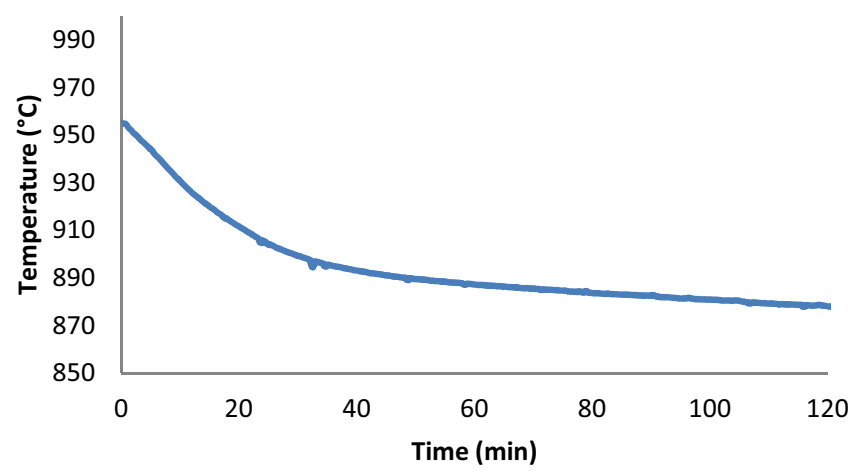

Fig. 6. Temperature of the PCM measured using a thermocouple at the bottom of the crucible.

holder, to prevent the oxidation of the carbon crucible. The heating speed is fixed at $5{ }^{\circ} \mathrm{C} \cdot \mathrm{min}^{-1}$.

2. The heating process imposes the use of an insulation block below the furnace. It decreases the heat flow from the crucible, thus reducing the heating time, and protects the exposed surface of the crucible from air oxidation. Indeed, a nitrogen flow rate of 16 L.min ${ }^{-1}$ (50 psi) is injected between the crucible and the insulation block in order to minimize air stagnation in this critical part of the crucible.

3. The PCM is completely melted after $4 \mathrm{~h}$ to $1000^{\circ} \mathrm{C}$; the steady state is then reached.

4. To initiate the cooling process, the insulation block is removed. A reference measurement is taken with the US device. No solid PCM is formed at this moment. The brass nozzle is then placed under the crucible, and a forced nitrogen flow is maintained. It is gradually increased from 0 up to $150 \mathrm{~L} \cdot \mathrm{min}^{-1}$, with $20 \mathrm{~L} \cdot \mathrm{min}^{-1}$ step increases to avoid any possible thermal shock. The cooling process is illustrated in Figure 6.

5. US measurements are done every $10 \mathrm{~min}$. At the same time, LVDT manual measurements, as proposed by Bertrand et al. [5], are also taken for comparison purposes.

When 3 successive LVDT measurements are within $5 \%$ difference, the bath is considered as totally solidified, and heating elements are stopped. The nitrogen flow is maintained until temperature falls below $450{ }^{\circ} \mathrm{C}$, at which crucible oxidation can be neglected.

\section{Experimental results}

Measurement results during a complete cooling session of PCM, both from LVDT and US devices, using the experimental method described in the previous section, are presented in Figure 7.

The deviation between US and reference LVDT measurements is relatively low and the proposed method allows proper monitoring of the ledge thickness during a complete cooling session, from the initial condition of complete liquid PCM to the final condition of complete solid PCM in the crucible. 


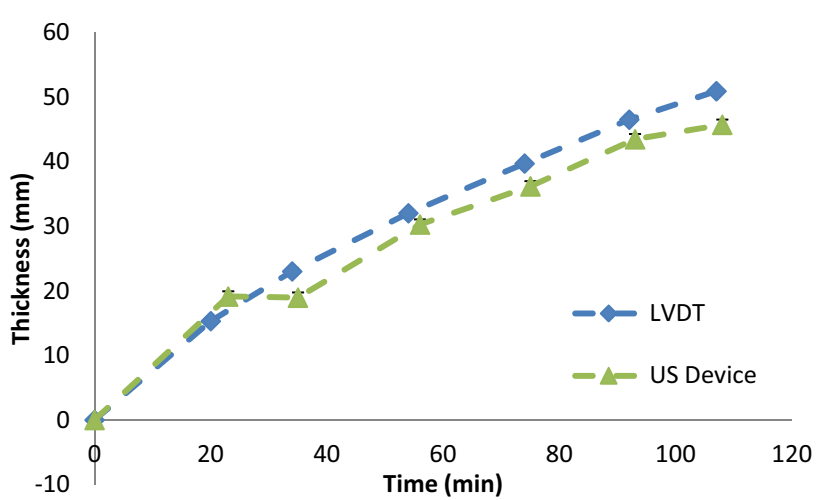

Fig. 7. US and LVDT thickness measurements during a complete cooling operation.

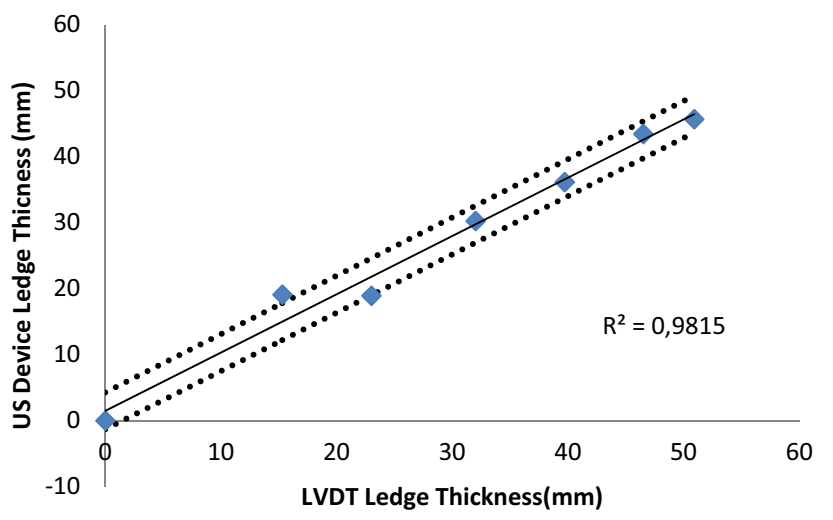

Fig. 8. Linear regression and $95 \%$ trust interval of US measurement.

A preliminary statistical analysis is proposed in Figure 8 to evaluate the precision of the US measurement.

Based on the available experimental results, a linear regression has been calculated. The standard deviation $\sigma$ has been calculated. A $95 \%$ trust interval is indicated with dotted lines, corresponding to $\pm 2 \sigma$, i.e. $\pm 2.8 \mathrm{~mm}$. The linear regression $R^{2}$ coefficient is 0.9815 , with only two points outside but close to the confidence interval. Based on this preliminary analysis, the risk of misevaluation of ledge thickness seems reduced, and the concept looks promising with an error below $10 \mathrm{~mm}$.

In addition, the crucible bottom thickness can also be evaluated using US method. According to the measurements (see Fig. 9), the carbon crucible thickness appears to decrease during the cooling process due to parasitic air oxidation. Assuming that the consumption of the crucible is linear with time, we can estimate the rate of consumption and compare it with the US measurements. Figure 9 compares the evolution of the crucible bottom thickness based on linear consumption to the measurements obtained with the US device.

The estimated crucible consumption is indicated with dotted lines. US measurements follow the trend of the thickness evolution of the crucible, despite of the measurements at 35 and 55 min, which are out of range. This is another indication that the US concept measurement of ledge thickness is efficient.

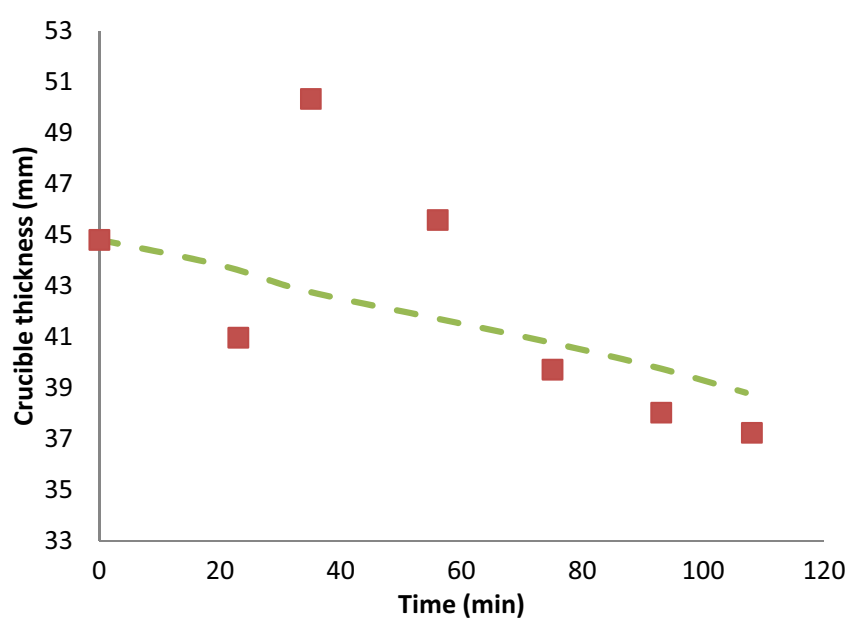

Fig. 9. Crucible graphite consumption estimated (dotted line) and measured by US device (points).

\section{Discussion and future developments}

Some observations can be notified according to the tests results. In Figure 6, two cooling speeds can be noticed. Indeed, during the first $20 \mathrm{~min}$. of cooling, the temperature decreases quickly, then, the temperature variation slows down due to the additional thermal resistance offered by the solid PCM growth. Those two stages are correlated with the two slopes found on the solid PCM thickness versus time curves shown in Figure 7. During higher cooling period, US measurements seem to well estimate the PCM thickness, considering the LVDT measurements as the reference measurement. When solidification speed decreases, i.e. for cooling time above 20 min, US measurements underestimate the ledge thickness. This can be attributed to the solidification characteristics of salt mixtures, which are typically occurring over a temperature range of $10-20^{\circ} \mathrm{C}$. Indeed, as reported by Lebreux et al. and by Marois et al. [17,18], a mushy zone is present in the solidification zone and the reflection induced by this soft interface can be more difficult to detect using US method.

Moreover, based on a qualitative evaluation of the sensor conditions after the experiments, we conclude that the design resisted to the high temperature conditions and that longer expositions should be performed to assess its robustness. In fact, the US device is still in perfect conditions, ready to be used for another set of measurements, despite being exposed to temperature up to $950{ }^{\circ} \mathrm{C}$. We conclude that the proof of concept of this new application of US technique in harsh conditions has been successfully performed.

Further developments include US measurements optimization, post-treatment automatization, and application of the instrumentation to industrial conditions that prevail in aluminum reduction plants in order to determine if this type of measurement could replace the mechanical probe actually used. Another exploration path comes along a complementary use of the acoustic emission probe. Indeed, during the solidification of the ledge, 
the transducer could be used solely as a receiver to detect acoustic emission changes induced by the modification of the ledge thickness. The authors expect that, as the ledge thickness increases, the bottom of the crucible becomes acoustically insulated. If the ambient noise inside of the crucible is powerful enough and transmitted in the transducer frequency range, the transducer could thus be used to determinate the ledge thickness as it is related to the signal attenuation.

\section{Conclusion}

The aim of the present work was to develop an original ultrasonic device able to perform a non-intrusive thickness measurement of the solidification front found inside metallurgical reactors such as aluminum electrolysis cells. The measurement environment and the experimental setup led to an adapted concept of an ultrasonic device composed of two transducers, an emitter and a receiver thermally protected by a quartz cylinder. Tests have been performed on an experimental setup mimicing the thermal environment of a real industrial metallurgical reactor. Results provided a good correlation between the proposed method and direct invasive measurement using a LVDT probe, within an accuracy range of $10 \mathrm{~mm}$. This innovative technique appears attractive for metallurgical industrial reactors because it does not require any complex numerical model nor intensive calculation for the on-line estimation of the PCM thickness. Hence, the aim of future work will be to experiment this new ultrasonic technique in real conditions of an industrial cell.

Acknowledgements. The authors would like to thank the R\&D team of Rio Tinto Alcan for the discussions and the logistical support. Moreover, the authors are grateful to the Fonds Québécois de la Recherche sur la Nature et les Technologies (FQRNT) for their financial support. Many thanks to François Allard for his appreciated help during the tests, and to the Groupe d'Acoustique de l'Université de Sherbrooke for the US equipment loan.

\section{References}

[1] L. Castonguay, M. Dupuis, Simulation of Kitimat VS dry anodes with the ANSYSßfinite element model, Rapport technique, Alcan International Ltd, 1986

[2] F.J. Stevens, Energy balance and cell dynamics: considerations for cell design, operations and process control, Proceedings of the 6th Australasian Aluminum Smelting Workshop, Thomastown, Australia, 1998, pp. 289-320

[3] K. Grjotheim, H. Kvande, Introduction to Aluminium Electrolysis, Understanding the Hall-Héroult Process, Aluminium-Verlag, Düsseldorf, 1993

[4] K.A. Rye, T. Eidet, K. Torklep, Dynamic ledge response in Hall-Heroult cells, TMS Light Metals 1999, San Diego, USA, 1999, pp. 347-352
[5] C. Bertrand, M. Désilets, G. Soucy, Experimental measurements within a phase change metallurgical reactor, Rev. Sci. Instrum. 82 (2011) 123902

[6] C. Bertrand, M.-A. Marois, M. Désilets, G. Soucy, M. Lacroix, A combined 2D inverse predictions and experimental analysis for the bank formation inside a metallurgical reactor, Int. J Heat Mass Trans. 59 (2013) 58-65

[7] M.-A. Marois, M. Désilets, M. Lacroix, Prediction of the Bank Formation in High Temperature Furnaces by a Sequential Inverse Analysis with Overlaps, Numer. Heat Transfer, Part A 60 (2011) 561-579

[8] M.J. Banet, M. Fuchs, J.A. Rogers, J.H. Reinold, J.M. Knecht, M. Rothschild, R. Logan, A.A. Maznec, K.A. Nelson, High-precision film thickness determination using a laser-based ultrasonic technique, Appl. Phys. Lett. 73 (1998) 169-171

[9] M. Falkenstrom, M. Engman, E. Lindh-Ulmgren, B. Hutchinson, Laser ultrasonics for process control in the metal industry, Non Destr. Test. Eval. 26 (2011) 237-252

[10] S. Dixon, C. Edwards, S.B. Palmer, High accuracy noncontact ultrasonic thickness gauging of aluminium sheet using electromagnetic acoustic transducers, Ultrasonics 39 (2001) 445-453

[11] T.E. Gomez Alvarez-Arenas, Simultaneous determination of the ultrasound velocity and the thickness of solid plates from the analysis of thickness resonances using aircoupled ultrasound, Ultrasonics 50 (2010) 104-109

[12] S. Dixon, B. Lanyon, G. Rowlands, Coating thickness and elastic modulus measurement using ultrasonic bulk wave resonance, Appl. Phys. Lett. 88 (2006) 141907-1

[13] L. Jakevicius, J. Butkus, A. Vladisauskas, Measurement of thickness of layer and sound velocity in multi-layered structure by the use of angular ultrasonic transducers, Ultragarsas 58 (2006) 20-24

[14] Y.H. Kim, S. Song, J. Lee, Simultaneous measurements of the ultrasonic wave velocity and thickness of a solid plate made from one side of the plate, Meas. Sci. Technol. 14 (2003) 13-16

[15] E.C. Brown, L. Mulvaney-Johnson, P.D. Coates, Ultrasonic measurement of residual wall thickness during gas assisted injection molding, Polym. Eng. Sci. 47 (2007) 1730-1739

[16] F.B. Cegla, J. Allin, J.O. Davies, P. Collins, P. Cawley, Ultrasonic wall thickness monitoring at high temperatures (500 C), Review of Progress in Quantitative Non destructive Evaluation 1335 (2011) 1325-1332, American Institute of Physics, USA

[17] M. LeBreux, M. Désilets, M. Lacroix, Fast Inverse Prediction of Phase Change Banks in High Temperature Furnaces with a Kalman Filter coupled with a Recursive Least-Square Estimator, Int. J. Heat Mass Trans. 53 (2010) 5250-5260

[18] M.-A. Marois, M. Désilets, M. Lacroix, Prediction of a 2D Solidification Front in High Temperature Furnaces by an Inverse Analysis, Numer. Heat Transfer, Part A 59 (2011) $151-166$ 